\title{
Input and output singularities for parallel manipulators
}

\author{
S.Vahid Amirinezhad and Peter Donelan \\ Victoria University of Wellington, Wellington 6012, New Zealand, \\ vahid.amirinezhad@sms.vuw.ac.nz, peter.donelan@vuw.ac.nz
}

\begin{abstract}
We develop a differential-geometric approach to kinematic modelling for manipulators which provides a framework for analysing singularities for forward and inverse kinematics via input and output mappings defined on the manipulator's configuration space.
\end{abstract}

Keywords: kinematic constraint mapping, configuration space, singularities

\section{Introduction}

The goal of understanding singularities of manipulators, especially parallel manipulators, has been a major challenge in robot kinematics for more than 35 years. While there is now detailed understanding of singularities for many specific architectures and a number of approaches to analysing and classifying singularities in general, there is not a robust mathematical model underpinning these. Major contributions are due to Gosselin and Angeles [6], Zlatanov et al [12] and Park and Kim [8], who provide a good review of research up to that time.

Following a method proposed by Piipponnen and Tuomela [9], the authors have developed a differential geometric approach to defining the configuration space (C-space) for a manipulator, based solely on constraints imposed on component links by the joints $[1,2]$. This approach, called the kinematic constraint mapping (KCM), demonstrates that, for a given family of manipulators, typically there is a single Grashof-type condition, expressible in terms of design parameters, for which the C-space has a singularity [3].

In this paper, we develop the theory to incorporate inputs and outputs, based on choice of actuated joints (inputs) and on the manipulator's workspace or end-effector (outputs). This approach has a number of advantages over many previous formulations:

(i) The roles of the variables describing link positions-pose variables in our terminology — and joint variables are clearly distinguished. This distinction has been blurred because often joint variables act as surrogates for a pose variables or vice versa.

(ii) The analysis of singularities is global - the definition of the C-space captures all configurations of all the links in the manipulator in terms of its pose 
variables, so singular configurations are explicitly identified. Rather than treating Jacobians as purely local realisations of the instantaneous kinematic relationship between joint velocities and link velocities, they are matrices of partial derivatives of globally defined mappings.

(iii) The KCM approach works for serial and parallel manipulators. It does not require inclusion of "loop-closure" constraints, which are simply corollaries of the joint constraints. As a result, it is easy to see why, for example, the forward kinematics of a serial mechanism are well defined.

In Section 2 we summarise the C-space definition, then in Sections 3 and 4 the definitions of joint variables, input and output mappings are introduced. Examples are then developed in Section 5 that demonstrate the efficacy of the approach. We conclude with some comments about forward and inverse kinematics.

\section{Configuration spaces}

We summarise briefly the KCM approach described in [1]. For a given manipulator, assign a reference coordinate frame $R$ and body coordinate frames $M_{i}$ for each link $i=1, \ldots, k$ (the number of links). The pose of each link is given by an element of the Euclidean group $S E(m),(m=2,3$ in planar and spatial cases, respectively). The coordinate frames determine isometries $S E(m) \cong S O(m) \times \mathbb{R}^{m}$. In the planar case, three pose variables $\mathbf{u}_{i}=\left(\theta_{i}, x_{i}, y_{i}\right)$ suffice to describe a pose while in the spatial case locally six parameters suffice, though dual quaternions may be used, along with additional equations, globally.

Each joint $J_{j}, j=1, \ldots, t$ (the number of joints), connecting links $L_{j_{-}}, L_{j_{+}}$, determines constraints on the corresponding pose variables that may be written in the form $f_{j}\left(\mathbf{u}_{j_{-}}, \mathbf{u}_{j_{+}}\right)=0 \in \mathbb{R}^{r_{j}}$, where $r_{j}$ is the number of constraints imposed by the joint. For example, for a planar revolute joint $r_{j}=2$; for a spatial spherical joint $r_{j}=3$. Each $f_{j}$ is termed a joint constraint mapping.

In addition to these joint constraints, it is usually assumed that one link is fixed as the base. This corresponds to adding an additional constraint mapping $f_{0}: S E(m) \rightarrow S E(m)$ where $f_{0}\left(\mathbf{u}_{b}\right)=$ const. In this case, $r_{0}=d_{m}:=$ $\operatorname{dim} S E(m)=\frac{1}{2} m(m+1)$. No additional constraints are required for loop closures: such constraints arise as a linear combination of joint constraint mappings.

Setting $s=\sum_{j=0}^{t} r_{j}$, the kinematic constraint mapping (KCM) is the function $F: S E(m)^{k} \rightarrow \mathbb{R}^{s}$, defined by $F=\left(f_{1}, \ldots, f_{t}\right)$ and the configuration space (C-space) is $\mathcal{C}=F^{-1}\left(0_{s}\right)$. Note that the functions $f_{j}$ may also involve design parameters (e.g. Denavit-Hartenberg parameters). For specific choices of parameters the KCM $F$ may be singular for some $\left(\mathbf{u}_{1}, \ldots, \mathbf{u}_{k}\right) \in \mathcal{C}$, which is to say its Jacobian matrix at that point has rank deficiency. This is called a $C$-space singularity. From now in we will restrict to the non-singular cases, so that $\mathcal{C}$ is a manifold of dimension $\mu=k d_{m}-s$ where $d_{m}$. 


\section{Joint variables}

In classical manipulator kinematics, joint variables, parametrising the freedom of displacement of one link with respect to another to which it is joined, play a critical role. Instantaneously, this freedom can be represented in terms of twistselements of the Euclidean Lie algebra $\mathfrak{s e}(m)$. The freedoms of a joint are the reciprocals of the (instantaneous) constraints with respect to the Klein form [10]. Globally, the relative motion between joined links is a coset of the subgroup of $S E(m)$ generated by the screws; in the case of 1-dof joints these are cosets of the 1-parameter subgroups $\exp (\phi X)$ where $X \in \mathfrak{s e}(m)$ and $\phi$ is the associated joint variable.

Let $f: S E(m) \times S E(m) \rightarrow \mathbb{R}^{r}$ be a joint constraint mapping. Since the pair of links (assuming no other constraints) is free to move without any relative displacement, for any $\left(g_{1}, g_{2}\right) \in f^{-1}(0) \subseteq S E(m) \times S E(m)$ and any $h \in S E(m)$, $f\left(h \circ g_{1}, h \circ g_{2}\right)=0$; that is, the joint constraint manifold is invariant under the action of the Euclidean group. In particular, we may take $h=g_{1}^{-1}$, so that the first component can be chosen as the identity $e \in S E(m)$. Given $(e, g) \in f^{-1}(0)$, the kernel of the Jacobian $J f(e, g)$ is a subspace of $\mathfrak{s e}(m) \times T_{g} S E(m)$. The subspace in the second component is the right translate by $g$ of a subspace of $\mathfrak{s e}(m)$ and a basis of twists determines the freedom of the joint.

In practice, joint variables can be realised as functions in terms of the pose variables for the joined links. For a planar revolute joint, the joint variable is the relative rotation $\phi_{j}$ between links and is given by the difference of the bodies' orientations with respect to the reference frame $R$ :

$$
\phi_{j}:=\theta_{j^{+}}-\theta_{j^{-}}
$$

The motion of link $j^{+}$, resulting from the freedom of this joint alone, has the form $\exp \left(\phi_{j} X\right) \circ g$ where $X$ is the twist representing rotation about the joint and $g$ represents the pose of link $j^{-}$.

For a planar prismatic joint, the variable is the relative translation $\phi_{j}$ between the links in the direction of the joint, given by the 2-norm of the difference between the links' positions with respect to the reference frame $R$

$$
\phi_{j}:=\left(\left(x_{j^{+}}-x_{j^{-}}\right)^{2}+\left(y_{j^{+}}-y_{j^{-}}\right)^{2}-d^{2}\right)^{1 / 2}
$$

where $d$ is a design parameter [2]. In practice, it is both sufficient and easier to work with norm squared $\phi_{j}^{2}$.

\section{Actuator space, workspace and input/output maps}

For a given manipulator, there may be several possible choices of actuated joints or inputs. Each of these may be represented by the associated joint variable. While, mathematically, any joint variable could be used, practical considerations reduce the choices. Similarly, there may be different choices for the end-effector 
or output. Once again, for a typical parallel manipulator, having a platform connected to a base by several serial legs, the only reasonable output is the configuration of the platform.

Suppose a set of $\lambda$ joints, each with just 1-dof, are selected to be actuated. We assume $\lambda \leq \mu=\operatorname{dim} \mathcal{C}$ allowing for under-actuation $(\lambda>\mu$ would result in constraints on the freedoms of actuated joints). The actuator space (A-space) $\mathcal{A}$ is the space parametrised by the actuated joint variables $\phi_{1}, \ldots, \phi_{\lambda}$, also called inputs.

For a given choice of end-effector or output link, the workspace (W-space) $\mathcal{W}$ is parametrised by the end-effector pose variables, which are called outputs. Note that $\nu:=\operatorname{dim} \mathcal{W} \geq \mu$; we allow inequality since the outputs may not be able to be parametrised by exactly $\mu$ pose variables.

Associated with these two spaces are functions defined on the manipulator $\mathrm{C}$-space. The input map $\pi_{a}: \mathcal{C} \rightarrow \mathcal{A}$ is defined by:

$$
\pi_{a}\left(\mathbf{u}_{1}, \ldots, \mathbf{u}_{k}\right)=\left(\phi_{1}, \ldots, \phi_{\lambda}\right)
$$

The output map $\pi_{w}: \mathcal{C} \rightarrow \mathcal{W}$ is the projection of $\mathcal{C}$ onto the space of pose variables for the end-effector. The overall model is illustrated in Fig. 1. In addition to the input and output maps, the relationships in each direction between inputs and outputs, denoted $\rho, v$, are the forward and inverse kinematic problems (FKP/IKP) discussed briefly in Sect. 6 .

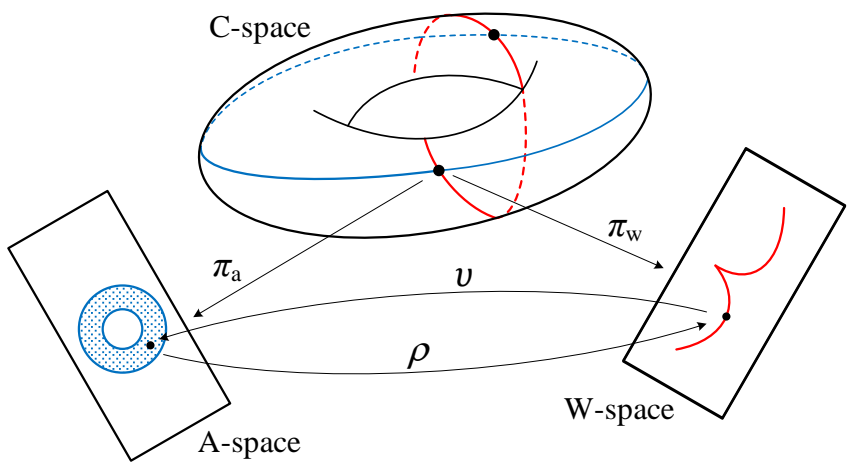

Fig. 1: Kinematic mappings and the $\mathrm{C}$-space

In order to detect input and output singularities, we apply an extension of the method of constrained optimisation which we state here in terms of mappings between Euclidean spaces but which generalises to smooth manifolds.

Theorem 1. Let $F: \mathbb{R}^{m} \rightarrow \mathbb{R}^{r}(m>r)$ and suppose that $\mathbf{0} \in \mathbb{R}^{r}$ a regular value so that $\mathcal{C}:=F^{-1}(\mathbf{0})$ is a smooth manifold of dimension $\mu=m-r$. Given a differentiable function $g: \mathbb{R}^{m} \rightarrow \mathbb{R}^{p}(p \geq \mu)$ then $\mathbf{u} \in \mathcal{C}$ is a singular point of $\left.g\right|_{\mathcal{C}}: \mathcal{C} \rightarrow \mathbb{R}^{p}$ if and only if rank $J_{\mathbf{u}}(g, F)<m$. 


\section{$5 \quad$ Examples}

We illustrate input/output maps with two planar parallel manipulators, as shown in Fig. 2. The results we derive are not new from a geometric point of view; see, for example $[5,11]$. However, they illustrate this robust approach, and provide a coherent explanation of forward and inverse kinematic singularities for parallel manipulators.

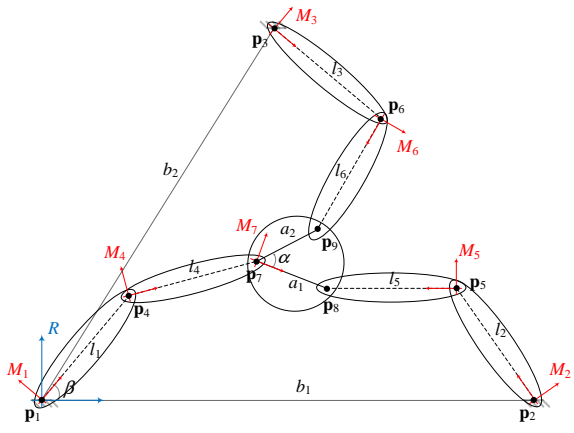

(a) 3-RRR PPM

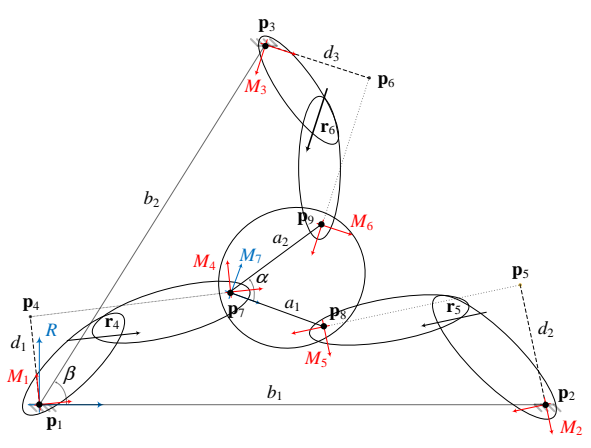

(b) 3-RPR PPM

Fig. 2: Parallel planar manipulators

The KCM approach to their C-spaces is given in $[1,2]$. The manipulators consist of $k=8$ rigid bodies, where we regard the ambient space as the fixed base. Consequently, we require $(8-1) \times 3=21$ pose variables $\left(x_{i}, y_{i}, \theta_{i}\right), i=1, \ldots, 7$, so that $\mathcal{C} \subset \mathbb{R}^{21}$; there are 12 design parameters in each case and away from a measure-zero subset of these, $\mathcal{C}$ is a manifold [3].

\subsection{3-RRR planar parallel manipulator}

The central link or platform, as shown in Fig. 2a, is classically chosen as the end-effector so that the workspace $\mathcal{W}$ is parametrised by $\left(x_{7}, y_{7}, \theta_{7}\right) \in \mathbb{R}^{3}$. Let $F: \mathbb{R}^{21} \rightarrow \mathbb{R}^{18}$ denote the KCM:

$$
\begin{aligned}
& \left(x_{i}, y_{i}, \theta_{i}\right)_{i=1, \ldots, 7} \mapsto \\
& \quad\left(-x_{1},-y_{1}, b_{1}-x_{2},-y_{2},-x_{3}+b_{2} c_{\beta},-y_{3}+b_{2} s_{\beta}, x_{1,4}+l_{1} c_{1}, y_{1,4}+l_{1} s_{1},\right. \\
& \quad x_{2,5}+l_{2} c_{2}, y_{2,5}+l_{2} s_{2}, x_{3,6}+l_{3} c_{3}, y_{3,6}+l_{3} s_{3}, x_{4,7}+l_{4} c_{4}, y_{4,7}+l_{4} s_{4}, \\
& \left.\quad x_{5,7}+l_{5} c_{5}-a_{1} c_{7}, y_{5,7}+l_{5} s_{5}-a_{1} s_{7} x_{6,7}+l_{6} c_{6}-a_{2} c_{\alpha, 7}, y_{6,7}+l_{6} s_{6}-a_{2} s_{\alpha, 7}\right)
\end{aligned}
$$

where $x_{i, j}=x_{i}-x_{j}, y_{i, j}=y_{i}-y_{j}, 1 \leq i<j \leq 7, c_{i}=\cos \theta_{i}, s_{i}=\sin \theta_{i}$, $i=1, \ldots, 7, c_{\alpha, 7}=\cos \left(\alpha+\theta_{7}\right), s_{\alpha, 7}=\sin \left(\alpha+\theta_{7}\right)$. Let $\hat{\pi}_{w}: \mathbb{R}^{21} \rightarrow \mathcal{W}$ denote projection onto the workspace, so that $\pi_{w}$ is the restriction of $\hat{\pi}_{w}$ to $\mathcal{C}$. To find the output singularities of 3 -RRR, we apply Theorem 1 , so consider $\Pi_{w}:=\left(\hat{\pi}_{w}, F\right)$ : $\mathbb{R}^{21} \rightarrow \mathbb{R}^{18} \times \mathbb{R}^{3}$ :

$$
\left(x_{i}, y_{i}, \theta_{i}\right)_{i=1, \ldots, 7} \mapsto\left(x_{7}, y_{7}, \theta_{7}, F\left(x_{1}, \ldots, \theta_{7}\right)\right) .
$$


The $21 \times 21$ Jacobian matrix $J \Pi_{w}$ can be reduced by deleting rows and columns of leading 1 s in row echelon form to a $9 \times 9$ matrix:

$$
J \Pi_{w}^{\mathrm{red}}=\left[\begin{array}{ccccccccc}
1 & 0 & 0 & 0 & 0 & 0 & 0 & 0 & 0 \\
0 & 1 & 0 & 0 & 0 & 0 & 0 & 0 & 0 \\
0 & 0 & 1 & 0 & 0 & 0 & 0 & 0 & 0 \\
-1 & 0 & 0 & -l_{1} s_{1} & 0 & 0 & -l_{4} s_{4} & 0 & 0 \\
0 & -1 & 0 & l_{1} c_{1} & 0 & 0 & l_{4} c_{4} & 0 & 0 \\
-1 & 0 & -a_{1} s_{7} & 0 & l_{2} s_{2} & 0 & 0 & l_{5} s_{5} & 0 \\
0 & -1 & a_{1} c_{7} & 0 & -l_{2} c_{2} & 0 & 0 & -l_{5} c_{5} & 0 \\
-1 & 0 & -a_{2} s_{\alpha, 7} & 0 & 0 & l_{3} s_{3} & 0 & 0 & l_{6} s_{6} \\
0 & -1 & a_{2} c_{\alpha, 7} & 0 & 0 & -l_{3} c_{3} & 0 & 0 & -l_{6} c_{6}
\end{array}\right]
$$

Its columns correspond to partial derivatives with respect to $x_{7}, y_{7}, \theta_{7}, \theta_{1}, \ldots, \theta_{6}$, and the top left $3 \times 3$ identity block matrix corresponds to the non-singular Jacobian of $\pi_{w}$. The bottom right $6 \times 6$ block matrix may be rank deficient if its determinant:

$$
l_{1} l_{2} l_{3} l_{4} l_{5} l_{6} \sin \left(\theta_{1}-\theta_{4}\right) \sin \left(\theta_{2}-\theta_{5}\right) \sin \left(\theta_{3}-\theta_{6}\right)=0 .
$$

Assuming design parameters $l_{i}>0 ; i=1, \ldots, 6$, equation ( 7 ) holds if $\theta_{i}=$ $\theta_{i+3}+\eta \pi ; i=1,2,3$, for some integer $\eta$, corresponding to a configuration in which one or more pair of leg links is collinear, as illustrated in Fig. 3 for the pair $l_{1}, l_{4}$.
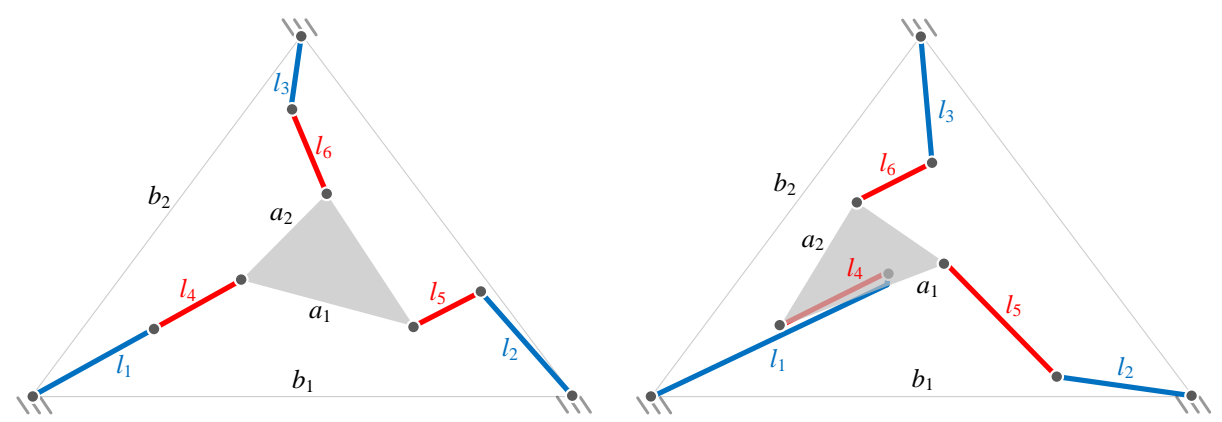

Fig. 3: 3-RRR output singularities

We now consider two distinct possibilities for the actuated joints: (a) the base anchor points and (b) the medial joints $\mathbf{p}_{4}, \mathbf{p}_{5}, \mathbf{p}_{6}$.

(a) $\mathbf{3}$ - $\underline{R} \mathbf{R}$. The three base joints $\mathbf{p}_{1}, \mathbf{p}_{2}, \mathbf{p}_{3}$ are the actuators. Identifying the base frame with the reference frame sets $\theta_{i^{-}}=0, i=1,2,3$ in equation (1). The joint variables are then given by $\phi_{i}=\theta_{i} ; i=1,2,3$ which span the A-space and the input map is the restriction to $\mathcal{C}$ of the projection:

$$
\hat{\pi}_{a}\left(x_{i}, y_{i}, \theta_{i}\right)_{i=1, \ldots, 7}=\left(\theta_{1}, \theta_{2}, \theta_{3}\right) .
$$


Setting $\Pi_{a}:=\left(\hat{\pi}_{a}, F\right): \mathbb{R}^{21} \rightarrow \mathbb{R}^{18} \times \mathbb{R}^{3}$, in a similar manner to the output map, the input singularities are given by the determinant of a $6 \times 6$ submatrix of the Jacobian:

$$
\operatorname{det}\left[\begin{array}{cccccc}
-l_{4} s_{4} & 0 & 0 & -1 & 0 & 0 \\
l_{4} c_{4} & 0 & 0 & 0 & -1 & 0 \\
0 & -l_{5} s_{5} & 0 & -1 & 0 & a_{1} s_{7} \\
0 & l_{5} c_{5} & 0 & 0 & -1 & -a_{1} c_{7} \\
0 & 0 & -l_{6} s_{6}-1 & 0 & a_{2} s_{\alpha 7} \\
0 & 0 & l_{6} c_{6} & 0 & -1 & -a_{2} c_{\alpha 7}
\end{array}\right]=l_{4} l_{5} l_{6}\left(a_{1} \sin \left(\theta_{4}-\theta_{6}\right) \sin \left(\theta_{5}-\theta_{7}\right)\right.
$$

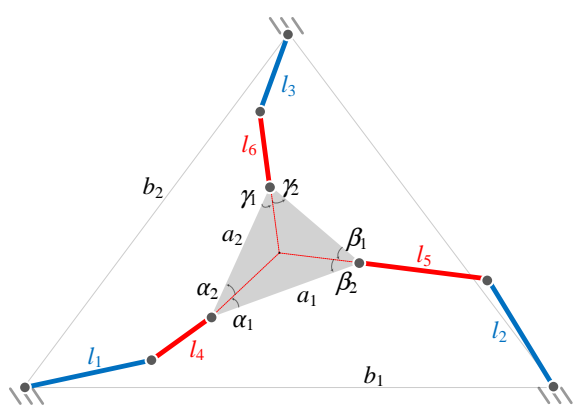

(a) $3-\underline{R R R}$

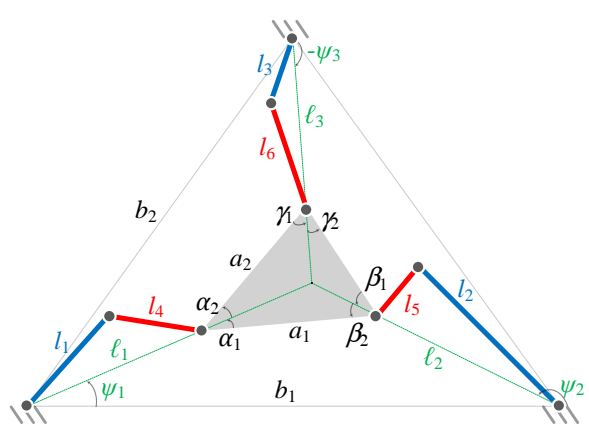

(b) $3-\mathrm{RRR}$

Fig. 4: 3-RRR input singularities

Given $l_{i}>0 ; i=4,5,6$, we employ a variant of Ceva's Theorem (see the Appendix) to interpret this geometrically. Comparison with equation (19), setting $\theta_{4}=\psi_{a}, \theta_{5}=\psi_{b}, \theta_{6}=\psi_{c}, \theta_{7}=\psi_{t}$, shows that this is equivalent to the lines spanned by links $l_{4}, l_{5}, l_{6}$ being concurrent. The intersection can take place either internally or externally to the platform triangle, as in Fig. 4a. Special cases include when two links are collinear, or all three parallel and intersect at infinity.

(b) 3-RRR. Selecting the medial joints $\mathbf{p}_{4}, \mathbf{p}_{5}, \mathbf{p}_{6}$ to be actuated, their joint variables, as in equation (1), are $\phi_{i}=\theta_{i}-\theta_{i-3} ; i=4,5,6$ and the input map $\hat{\pi}_{a}$ is the restriction to $\mathcal{C}$ of:

$$
\left(x_{i}, y_{i}, \theta_{i}\right)_{i=1, \ldots, 7} \mapsto\left(\theta_{4}-\theta_{1}, \theta_{5}-\theta_{2}, \theta_{6}-\theta_{3}\right) .
$$

The resulting condition for input singularities reduces to:

$$
\operatorname{det}\left[\begin{array}{cccccc}
-l_{1} s_{1}-l_{4} s_{4} & 0 & 0 & -1 & 0 & 0 \\
l_{1} c_{1}+l_{4} c_{4} & 0 & 0 & 0 & -1 & 0 \\
0 & -l_{2} s_{2}-l_{5} s_{5} & 0 & -1 & 0 & a_{1} s_{7} \\
0 & l_{2} c_{2}+l_{5} c_{5} & 0 & 0 & -1 & -a_{1} c_{7} \\
0 & 0 & -l_{3} s_{3}-l_{6} s_{6}-1 & 0 & a_{2} s_{\alpha 7} \\
0 & 0 & l_{3} c_{3}+l_{6} c_{6} & 0 & -1 & -a_{2} c_{\alpha 7}
\end{array}\right]=0 .
$$


This closely resembles the matrix given in (9) except the terms in the first three columns denote the coordinates of the leg vectors connecting corresponding base and platform anchor points $\left(x_{i, i+3}, y_{i, i+3}\right)=\left(l_{i} c_{i}+l_{i+3} c_{i+3}, l_{i} s_{i}+l_{i+3} s_{i+3}\right), i=$ $1,2,3$. From Ceva's Theorem, the geometric interpretation of this condition is that these vectors intersect, see Fig. $4 \mathrm{~b}$.

\section{$5.2 \quad 3$-RPR planar parallel manipulator}

The 3-RPR PPM is depicted in Fig. 2b. As for the 3-RRR, the platform determines the workspace and the associated output mapping is in the form of (5). To find output singularities, we compute the Jacobian of $\Pi_{w}=\left(\hat{\pi}_{w}, F\right): \mathbb{R}^{21} \rightarrow$ $\mathbb{R}^{18} \times \mathbb{R}^{3}$ which, after reduction, simplifies to the condition:

$$
\left(x_{1,4} c_{1}+y_{1,4} s_{1}\right)\left(x_{2,5} c_{2}+y_{2,5} s_{2}\right)\left(x_{3,6} c_{3}+y_{3,6} s_{3}\right)=0 .
$$

The vectors $\mathbf{v}_{i}=\left(x_{i, i+3}, y_{i, i+3}\right), i=1,2,3$ connect the revolute joints in each leg, while $\mathbf{u}_{i}=\left(c_{i}, s_{i}\right)$ designate (unit) directions of the prismatic joints $\mathbf{r}_{i+3}, i=$ $1,2,3$. Hence, an output singularity occurs whenever $\xi_{i}:=\mathbf{v}_{i} \cdot \mathbf{u}_{i}=0$, i.e. one of the $\mathbf{v}_{i}$ is orthogonal to the corresponding joint direction or vanishes (so that base and anchor joints coincide). We consider two options for actuated joints.

(a) 3-RPR. Choosing the base revolute joints $\mathbf{p}_{1}, \mathbf{p}_{2}, \mathbf{p}_{3}$ as actuators, as in Sect. 5.1 the actuated joint variables are $\phi_{i}=\theta_{i} ; i=1,2,3$. Applying Thm. 1 , the $21 \times 21$ Jacobian matrix can be reduced so that input singularities are determined by the $3 \times 3$ determinant:

$$
\operatorname{det}\left[\begin{array}{ccc}
-s_{1} & c_{1} & 0 \\
-s_{2} & c_{2} & -a_{1} c_{2,7} \\
-s_{3} & c_{3}-a_{2} c_{3,7, \alpha}
\end{array}\right]=\begin{gathered}
a_{1} \sin \left(\theta_{1}-\theta_{3}\right) \sin \left(\theta_{2}-\theta_{7}+\frac{\pi}{2}\right) \\
\quad+a_{2} \sin \left(\theta_{1}-\theta_{2}\right) \sin \left(\alpha-\theta_{3}+\theta_{7}+\frac{\pi}{2}\right)=0 .
\end{gathered}
$$

This corresponds to Ceva's condition (19), where $\psi_{a}=\theta_{1}+\frac{\pi}{2}, \psi_{b}=\theta_{2}+\frac{\pi}{2}, \psi_{c}=$ $\theta_{3}+\frac{\pi}{2}, \psi_{t}=\theta_{7}$. Geometrically, the lines through the platform anchor points and orthogonal to the prismatic joints are concurrent.

(b) 3-RPR. Actuating the prismatic joints $\mathbf{r}_{4}, \mathbf{r}_{5}, \mathbf{r}_{6}$, so $\phi_{i}, i=4,5,6$ as given in equation (2), are joint variables, the input mapping can be taken as:

$$
\left(x_{i}, y_{i}, \theta_{i}\right)_{i=1, \ldots, 7} \mapsto\left(\left(x_{1,4}^{2}+y_{1,4}^{2}\right),\left(x_{2,5}^{2}+y_{2,5}^{2}\right),\left(x_{3,6}^{2}+y_{3,6}^{2}\right)\right)
$$

Theorem 1 yields a $21 \times 21$ Jacobian matrix which can be reduced and simplified to give the determinant:

$$
\xi_{1} \xi_{2} \xi_{3}\left(a_{1} \sin \left(\psi_{1}-\psi_{3}\right) \sin \left(\theta_{7}-\psi_{2}\right)-a_{2} \sin \left(\psi_{1}-\psi_{2}\right) \sin \left(\alpha+\theta_{7}-\psi_{3}\right)\right)=0,
$$

where $\psi_{i}$ denote the angles of the legs with respect to the reference frame. Corresponding input singularities are given by (i) $\xi_{i}=0, i=1,2,3$, which are the output singularity conditions, or (ii) the vanishing of the bracket, which by Ceva's condition (19), corresponds to the extensions of the leg vectors being concurrent. 


\section{Conclusion: forward and inverse kinematics}

In much of the manipulator singularity literature the models have been encoded by an implicit function $F(\theta, \mathbf{x})=0$. The ability to express inputs $\theta$ or outputs $\mathbf{x}$ explicitly in terms of the other can generally only be solved locally and requires the conditions for the Implicit Function Theorem (see, for example, [7]) to be met. However, it is known that analysing this implicit function alone does not provide full information about manipulator singularities in the parallel case. As is clear from Fig. 1, the forward and inverse relations can be determined in terms of the input and output maps, provided these have well defined (local) inverses:

$$
\rho=\pi_{a}^{-1} \circ \pi_{w}, \quad v=\pi_{w}^{-1} \circ \pi_{a} .
$$

The existence of the inverses is a local question. Assume, for the moment, that $\operatorname{dim} \mathcal{A}=\operatorname{dim} \mathcal{C}=\operatorname{dim} \mathcal{W}$. Then $\pi_{a}$ or $\pi_{w}$ has a local inverse at some $\left(\mathbf{u}_{1}, \ldots, \mathbf{u}_{k}\right) \in \mathcal{C}$ if and only if the Jacobian at that point is non-singular. Therefore, the input and output singularities are critical to the solutions of the FKP and IKP. We note, for example, that for a serial manipulator, the C-space is isomorphic to the actuator space so that $\pi_{a}$ is always invertible and the FKP is well-posed.

\section{References}

1. Amirinezhad, S.V., Donelan, P.: Kinematic constraint maps, C-space singularities and generalised Grashof conditions. In: 2017 IDETC/CIE Conference. ASME (2017)

2. Amirinezhad, S.V., Donelan, P.: Kinematic constraint maps and c-space singularities for planar mechanisms with prismatic joints. In: International Symposium on Advances in Robot Kinematics. pp. 212-220. Springer (2018)

3. Amirinezhad, S.V., Donelan, P., Müller, A.: Transversality and its applications to kinematics. In: International Symposium on Advances in Robot Kinematics. pp. 221-229. Springer (2018)

4. Coxeter, H.S.M.: Introduction to geometry, vol. 136. Wiley New York (1969)

5. Daniali, H.M., Zsombor-Murray, P., Angeles, J.: Singularity analysis of planar parallel manipulators. Mechanism and Machine Theory 30(5), 665-678 (1995)

6. Gosselin, C., Angeles, J.: Singularity analysis of closed-loop kinematic chains. IEEE Journal of Robotics and Automation 6(3), 281-290 (June 1990)

7. Krantz, S.G., Parks, H.R.: The implicit function theorem: history, theory, and applications. Springer Science \& Business Media (2012)

8. Park, F.C., Kim, J.W.: Manipulability of closed kinematic chains. Journal of Mechanical Design 120, 542-548 (1998)

9. Piipponen, S., Tuomela, J.: Algebraic analysis of kinematics of multibody systems. Mechanical Sciences 4(1), 33-47 (2013)

10. Selig, J.M.: Geometric fundamentals of robotics. Springer Science \& Business Media (2004)

11. Yang, G., Chen, W., Chen, I.M.: A geometrical method for the singularity analysis of 3-RRR planar parallel robots with different actuation schemes. In: Intelligent Robots and Systems, 2002. IEEE/RSJ International Conference on. vol. 3, pp. 2055-2060. IEEE (2002) 
12. Zlatanov, D., Fenton, R.G., Benhabib, B.: Identification and classification of the singular configurations of mechanisms. Mechanism and Machine Theory 33(6), 743-760 (1998)

\section{Appendix: Ceva's Theorem}

This theorem can be found in many texts on Euclidean geometry, e.g. [4]. We state it here in terms of angle division, rather than the more common side division.

Theorem 2. Three lines $\ell_{a}, \ell_{b}, \ell_{c}$ passing through the vertices of a triangle $A B C$ and dividing the internal angles $\alpha, \beta, \gamma=\pi-\alpha-\beta$ into sub-angles $\left(\alpha_{1}, \alpha_{2}\right)$, $\left(\beta_{1}, \beta_{2}\right),\left(\gamma_{1}, \gamma_{2}\right)$ are concurrent if and only if:

$$
\sin \alpha_{1} \sin \beta_{1} \sin \gamma_{1}=\sin \alpha_{2} \sin \beta_{2} \sin \gamma_{2}
$$

We derive an equivalent condition in terms of the angles the lines and one side of the triangle make with respect to a given reference frame. Denote by $\psi_{t}$ the angle the side $A B$ makes with the $x$-axis of the reference frame and $\psi_{a}, \psi_{b}, \psi_{c}$ the angles lines $\ell_{a}, \ell_{b}, \ell_{c}$ make with the same axis, as shown in Fig. 5.

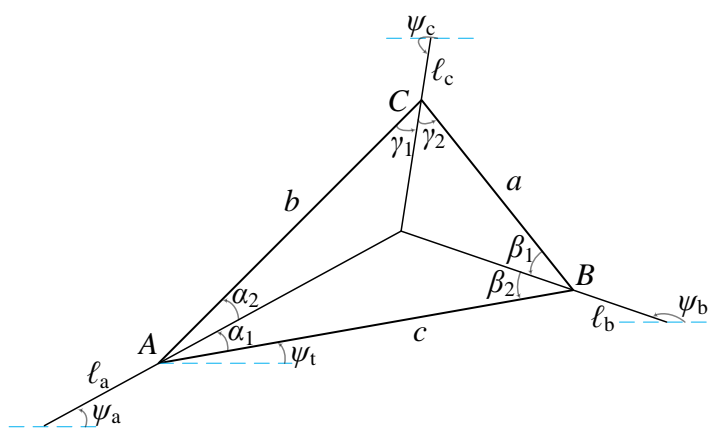

Fig. 5: Ceva's theorem

The six sub-angles $\alpha_{i}, \beta_{i}, \gamma_{i} ; i=1,2$ can be expressed in terms of $\psi_{a}, \psi_{b}, \psi_{c}, \psi_{t}$ and the given angles $\alpha, \beta, \gamma$ :

$$
\begin{array}{lll}
\alpha_{1}=\psi_{a}-\psi_{t}, & \beta_{1}=\beta-\pi+\psi_{b}-\psi_{t}, & \gamma_{1}=-\pi-\alpha+\psi_{c}-\psi_{t} \\
\alpha_{2}=\alpha-\psi_{a}+\psi_{t}, & \beta_{2}=\pi-\psi_{b}+\psi_{t}, & \gamma_{2}=2 \pi-\beta-\psi_{c}+\psi_{t}
\end{array}
$$

Substituting in equation (17), utilising trigonometric identities, and by the sine rule, $a_{1} \sin (\alpha+\beta)=a_{2} \sin \beta$ the condition can be restated as:

$$
\begin{aligned}
0= & \sin \left(\psi_{a}-\psi_{t}\right) \sin \left(\beta+\psi_{b}-\psi_{t}\right) \sin \left(\alpha-\psi_{c}+\psi_{t}\right) \\
& \quad-\sin \left(\alpha-\psi_{a}+\psi_{t}\right) \sin \left(\beta+\psi_{c}-\psi_{t}\right) \sin \left(\psi_{b}-\psi_{t}\right) \\
= & \sin (\alpha+\beta) \sin \left(\psi_{a}-\psi_{c}\right) \sin \left(\psi_{b}-\psi_{t}\right)-\sin (\beta) \sin \left(\psi_{a}-\psi_{b}\right) \sin \left(\alpha-\psi_{c}+\psi_{t}\right) \\
= & a_{1} \sin \left(\psi_{a}-\psi_{c}\right) \sin \left(\psi_{b}-\psi_{t}\right)-a_{2} \sin \left(\psi_{a}-\psi_{b}\right) \sin \left(\alpha-\psi_{c}+\psi_{t}\right)
\end{aligned}
$$


This is an Author Accepted Manuscript version of the following chapter: S. Vahid Amirinezhad \& P. Donelan, "Input and output singularities for parallel manipulators", published in Advances in Mechanism and Machine Science, IFToMM WC 2019, Mechanisms and Machine Science, vol 73, Springer, Cham, reproduced with permission of Springer Nature Switzerland. The final authenticated version is available online at https://doi.org/ 10.1007/978-3-030-20131-9_29 